\title{
Editorial
}

\section{Quality and Operations Management in Food Supply Chain}

\author{
Yong He $\mathbb{D}^{1},{ }^{1}$ Dong Li, ${ }^{2}$ Sarah J. Wu, ${ }^{3}$ and Chunming Shi ${ }^{4}{ }^{4}$ \\ ${ }^{1}$ School of Economics and Management, Southeast University, Sipailou 2, Nanjing 210096, China \\ ${ }^{2}$ Management School, University of Liverpool, Chatham Street, Liverpool L6972H, UK \\ ${ }^{3}$ The Gabelli School of Business, Fordham University, 441 E. Fordham Road, NY 10458, USA \\ ${ }^{4}$ Lazaridis School of Business and Economics, Wilfrid Laurier University, Waterloo, ON, Canada N2L 3C5
}

Correspondence should be addressed to Yong He; hy@seu.edu.cn

Received 8 March 2018; Accepted 8 March 2018; Published 13 May 2018

Copyright (C) 2018 Yong He et al. This is an open access article distributed under the Creative Commons Attribution License, which permits unrestricted use, distribution, and reproduction in any medium, provided the original work is properly cited.

Quality of food products changes continuously during various stages like transportation and storage, which brings challenges to the operations management of food supply chains. This special issue focuses on the quality and operations management issues in food supply chain management. The articles in this special issue provide important insights for firms to improve operations process control, reduce waste, lower cost, meet customers' expectation, or mitigate food safety risks. Y. He et al. present a literature review on quality and operations management problems in food supply chains. In the review, gaps are identified in this research area along with suggestions and directions for future research.

Evaluating quality risk levels in food supply chains can reduce quality information asymmetry, minimize food quality incidents, and promote integrated regulations for food quality. However, robust and quantitative methods are rarely reported for assessing the risk level of every link in food supply chains and evaluating the overall risk. As such, there is a need to provide effective guidance for the quality risk evaluation in the whole food supply chain. To address this problem, L. Bai et al. study the food supply chain using a quality risk evaluation indicator system. They develop a mathematical model based upon the fuzzy comprehensive evaluation model (FCEM) and failure mode, effects, and criticality analysis (FMECA) so as to evaluate the quality risk level in the food supply chain. They conduct computational experiments to verify the effectiveness and feasibility of this proposed model through a questionnaire survey.

Quality change affects customers' preferences for food products. To enhance their profitability, firms usually utilize behavior-based pricing strategies. Due to the lack of information about food freshness, consumers' decision-making highly depends on sales prices and manufacturing and expiry dates of items. P.-Y. Chen develops an EOQ model assuming that dealers are able to determine the sales price at each point of time and predict customers' intention of buying food items in varying freshness. As a result, dealers can set an optimal inventory cycle and allocate a weekly sales price for each time point to maximize the profit per unit time. This model allows dealers to discover the optimal sales prices and to identify the optimal solution for fresh goods retailers to conduct immediate price control. Since bundling strategy is an efficient way to reduce storage cost and to meet customer quality requirements, Y. Fang et al. establish a nonlinear mixed integer programming model to formulate the bundle pricing problem for fresh products, where they assume that two or more fresh products can be sold together in one united price.

Information asymmetry is prevalent in food supply chains. On the one hand, it affects pricing or inventory decisions. On the other hand, consumers could not identify the real quality until purchasing and consuming the experience goods. In some cases, they may not know the quality or variety even after consuming the credence goods such as genetically modified (GM) food. In the context of asymmetric information and scientific uncertainty, L. Zhao et al. explore pricing strategies between two firms producing horizontally and vertically differentiated foods. The type of a firm's product is not public information, but its pricing decision may signal its product type to consumers. Additionally, they consider the 
scientific uncertainty as a risk with a known distribution, the expected potential net damage, and the volatility of risk. Their study aims to combine market mechanisms with government regulations to separate GM food from the conventional food conforming to consumer's right to know. J. Wu et al. consider a food supply chain with two competing manufacturers and multiple competing unreliable suppliers (i.e., multisourcing). While the suppliers compete on price, the manufacturers compete on quantity. They develop a stylized multistage game theoretic model to examine the impact of key parameters, including the level of yield uncertainty, two manufacturers' cost correlation, the correlated coefficient of suppliers' yield processes, and the number of suppliers on the manufacturers' willingness to vertical cost-information sharing. Empirically, Y. Sun et al. conduct a survey of 450 agricultural product suppliers and obtain some interesting results by statistically analyzing the impact of antecedents on fairness perception and the influence of fairness perception on relationship quality. They also analyze moderating effect of dependence on the impact path from fairness perception to relationship quality (i.e., trust and commitment).

The $\mathrm{O} 2 \mathrm{O}$ (Online to Offline) business model has become more popular with the fast development of Internet and information technology. This business model has also been adopted by some food providers. X. Yu and X. Ren study a dual-selling model with an online food processing factory and an offline retailer under the impact of food quality information service. The study provides useful managerial implications to firms in the industry.

Overall, the above-mentioned studies significantly contribute to both literature and practice, which bridges the gaps in the research area of food supply chain management. We hope that readers of this special issue find the selected contributions interesting. We would be delighted if this special issue could serve as a strong driving force to push the future research on food quality and operations management in food supply chains to a higher level.

Yong He

Dong Li

Sarah J. Wu

Chunming Shi 


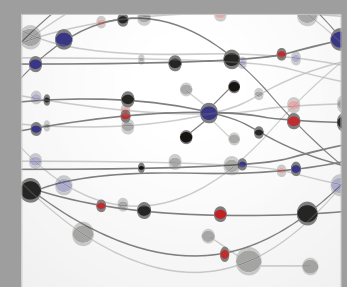

The Scientific World Journal
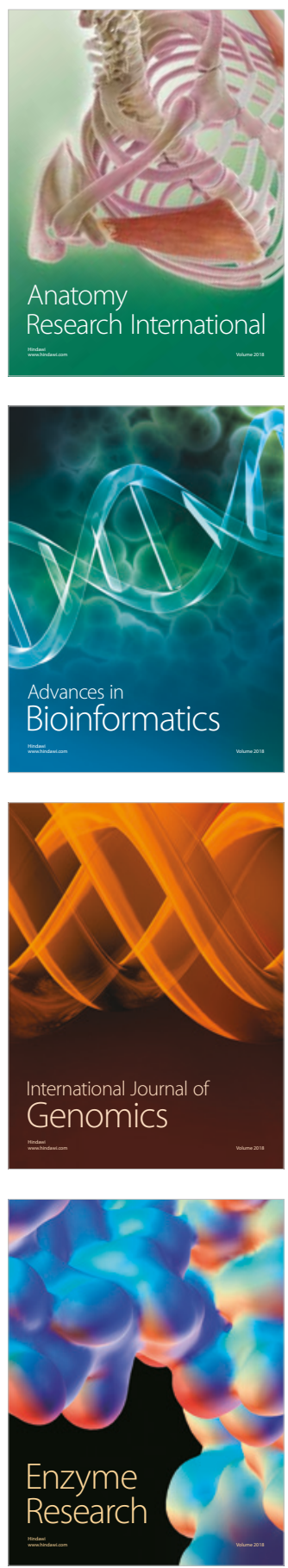
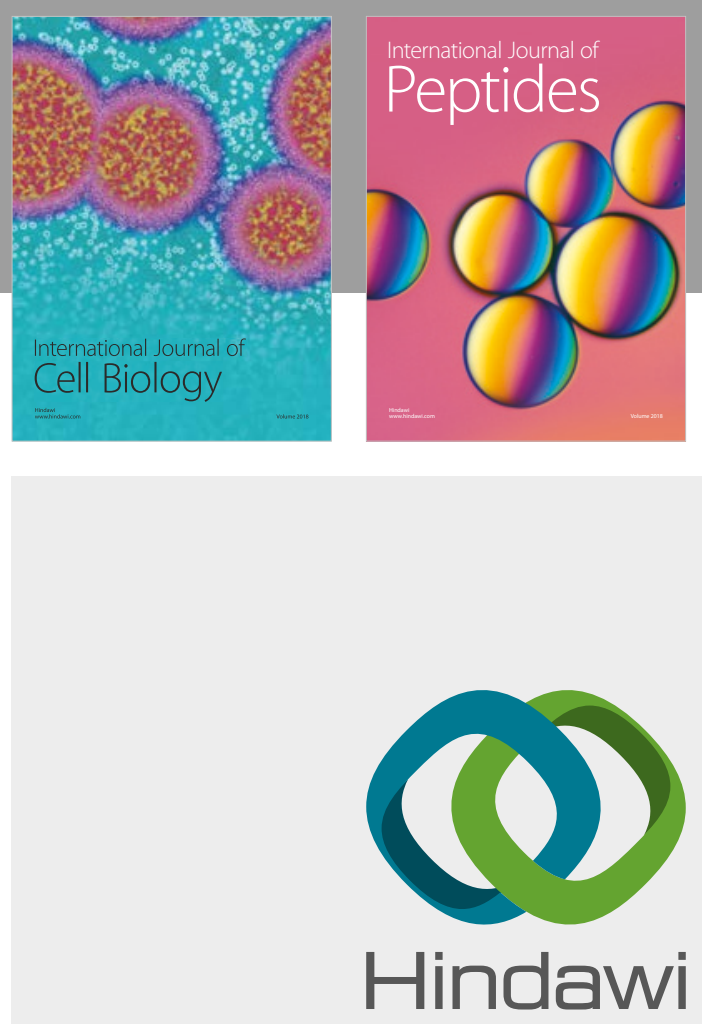

Submit your manuscripts at

www.hindawi.com
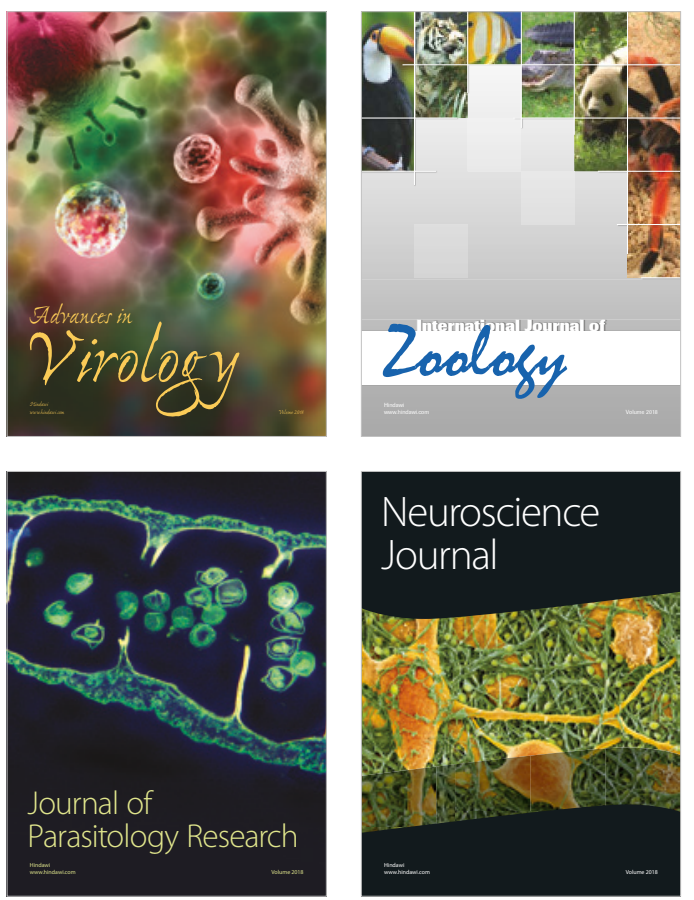
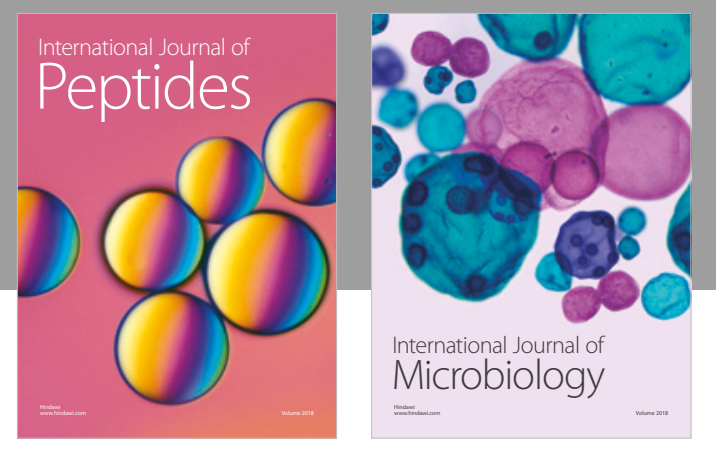

nternational Journal of Microbiology
Journal of
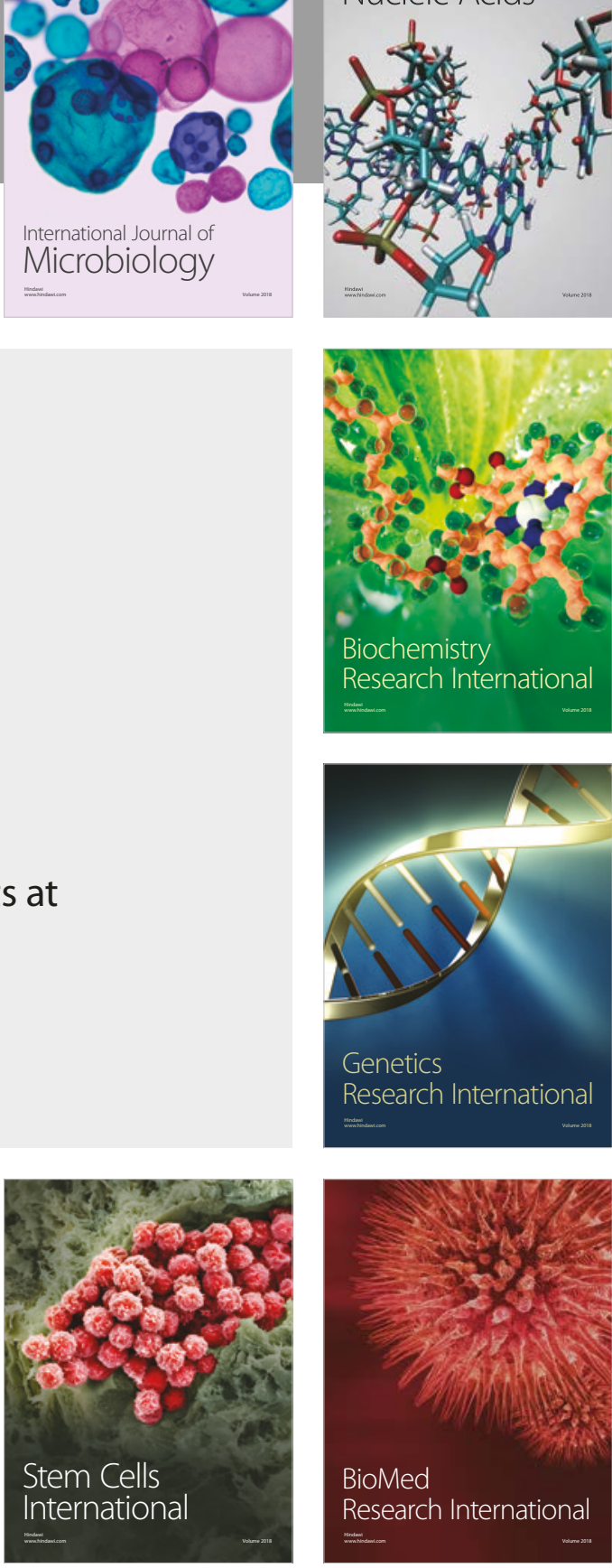
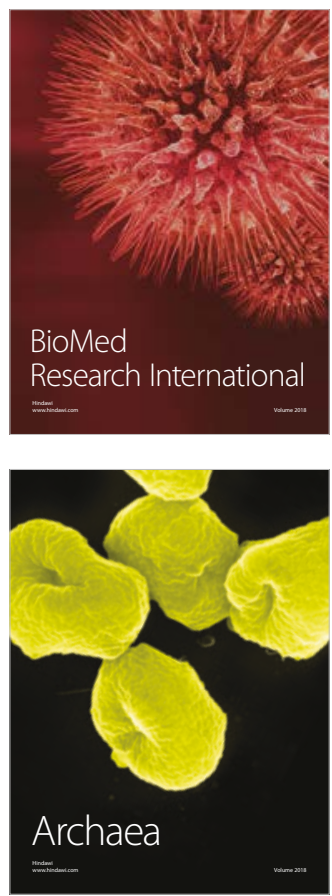